\title{
The development of obstetric anesthesiology in mainland China
}

\author{
Lisha Hu, MD, Shanglong Yao, MD, PhD \\ Department of Anesthesiology, Institute of Anesthesiology and Critical Care Medicine
}

Union Hospital, Tongji Medical College, Huazhong University of Science and Technology

\section{Abstract}

Obstetric anesthesiology is a significant component of modern anesthesiology, and a high risk sub-specialty because of its involvement of both mother and newborn. Having a late start, obstetric anesthesiology in mainland China has been developing synchronously with all other anesthesiology sub-specialties. Advances in science and technology and improvement of management skills in recent decades, resulted in the evolution of obstetric anesthesiology as an important subspecialty providing services to almost all aspects of obstetric services. While influenced by Chinese economic conditions and local policies, the development of obstetric anesthesiology in mainland China presents some unique features. With the implementation of the two-child policy in 2016, the number of pregnant women with advanced maternal age and potential scarred uterus will inevitably rise, potentially leading to higher incidence of placenta previa, placental abruption and other obstetric complications. These issues will subsequently pose challenges for anesthesia providers. Confronting the higher risks of obstetric anesthesia, we should utilize new technology and new management strategy more aggressively, strengthen our residency training, and keep abreast of the latest development in the specialty to maximize safety of obstetric patients. By increasing awareness of the obstetric risks for anesthetist/anesthesiologists, obstetrician and hospital administrators, emphasizing crisis management in obstetric services, building a comprehensive management team centering on patient care, applying the most advanced technology and optimizing the process and specifications of obstetric anesthesia and labor analgesia, will ultimately improve the quality of obstetric anesthesiology in mainland China.

Keywords: development, history, obstetric anesthesiology, China

Obstetric anesthesiology is a significant component of modern anesthesiology, and it is a high-risk subspecialty among other subspecialties of clinical anesthesia. Obstetric anesthesiology involves taking care of both mother and fetus/newborn [1]. Therefore, anesthesia providers should acquire not only thorough knowledge and skills of anesthesiology, but also overall understanding of physiologic and pathological changes of pregnancy and other obstetric disorders, effects of different drugs on mother and fetus/infant, and pathogenesis and management of various obstetric disorders. Obstetric anesthesia is obligated to provide maximal safety and the highest standard of care to both parturient and fetus/newborn, and offer reasonably comfortable process of labor and delivery. With the progress in technology and management strategies over the past years, obstetric anesthesiology in China is also developing rapidly, however, with a relatively late start which was adversely affected by its economic conditions, cultures, and local policies and regulations. The development of obstetric anesthesiology in mainland China still has a long way to go.

\section{The origin of obstetric anesthesiology}

Modern obstetric anesthesiology began with the emergence and development of anesthesiology. The beginning of clinical anesthesia can be traced back to Sir Humphry Davy who used nitrous oxide, the first anesthetic in 1799. On March 30, 1842, Dr. Crawford Long for the very first time in human history used sulfuric ether to anesthetize a patient for the removal of a tumor from the neck of a patient. And on October 16, 1846, Morton successfully used ether to provide anesthesia for a surgeon to remove a mandibular mass at the Massachusetts General Hospital. All these marked the beginning of clinical anesthesiology. In 1847, James Young Simpson used chloroform for childbirth-related pain management, which could be claimed as the beginning of obstetric anesthesia. Later in 1853, John Snow anesthetized Queen Victoria for delivery also with chloroform. In 1942, Robert Hingson applied epidural anesthesia to parturient for the first time, and achieved great relief of labor pain and postoperative pain. More than 100 years later in 1953, American physician established the Ap- 
gar scoring system, which provided a reliable standard for accurately evaluating the newborn and managing neonatal risk in timely fashion. APGAR Score contributed significantly to the improved survival of newborns and facilitated a rapid development of neonatology [1].

With a relatively late start, obstetric anesthesiology in mainland China as a subspecialty has been developing on pace with general anesthesiology in China. In 2011, the Chinese Society of Anesthesiology (CSA) established the Obstetric anesthesia committee, is committed to the overall development of obstetric anesthesia and improvement of the quality of obstetric anesthesia in mainland China, and aims to reduce complications and adverse effects related to obstetric anesthesia by establishing guidelines and regulations. The obstetric anesthesia committee invited many experts to set up standards, guidelines and consensus of obstetric anesthesia. The committee also designated more than 20 obstetric anesthesia training sites in mainland China. They organized and sponsored many intermediate and senior level professional training programs in hospitals of all levels, in attempt to train key personnel and improve the quality in obstetric anesthesia.

\section{The status of obstetric anesthesiology}

In recent years, obstetric anesthesiology in mainland China has been growing quickly. A great deal of progress has been made in the management of obstetric patients. Despite the late start, obstetric anesthesiology team has been designated in most major hospitals in mainland China, and the obstetric anesthesia team covers almost all key fields of obstetric practice including routine elective cesarean section, cesarean section of critically ill parturient, neonatal resuscitation, fetal surgery, analgesia after cesarean section, labor and delivery, and potentially intraoperative cell salvage in cesarean section [2-6]. It has achieved great outcomes in clinical practice and scientific research in the field of obstetric anesthesia, improved prognosis of mother and fetus/infant, and promoted the development of obstetrics in mainland China.

\section{Pregnant women with advanced age, scarred uterus and various complications}

According to population statistics in mainland China, the initial age of childbearing has been delayed from 22.8 in 1970 to 26.6 in 2007 . The cumulative fertility rate of women over 30 years old accounted for $30 \%$ of total fertility rate from 2000 to 2007 , up from $15 \%$, and this trend continues consistently. With the implementation of the two-child policy in 2016, this trend will likely accelerate, accompanied by a rising number of pregnant women with scarred uterus and higher incidence of placenta previa and placental abruption [7]. In addition, an increase of older pregnant women, the rate of hypertension and coronary artery disease, the percentage of premature birth and death, and the dramatic increase in obese pregnant women, leads to increased difficulty in neuraxial anesthesia placement and management [8]. By close monitoring of these patients, we can have early detection of problems, appropriate selections of anesthesia methods, drugs and devices, and aggressive management. The above-mentioned highrisk parturient can also be offered good quality of obstetric anesthesia services with good clinical outcomes.

\section{General anesthesia in caesarean section}

The first anesthetic choice for cesarean section is usually neuraxial anesthesia [9]. If parturient has contraindications for neuraxial anesthesia, then general anesthesia will be the alternative option, though local anesthetic infiltration and intravenous sedation can also be a choice. The latter usually won't provide complete anesthesia for cesarean section and patients may have poor perioperative experience, thus it may potentially affect the prognosis of the newborn [10]. As new drugs and new anesthetic techniques continuously improve, rational selections of shorter-acting, rapid-onset drugs will have significantly less impact on mother and fetus/newborn, thus better ensuring the safety of parturient undergoing cesarean section.

\section{Management of pregnancy with heart disease}

During pregnancy, especially middle and late stage of pregnancy, pregnant women undergo dramatic hemodynamic alterations [11]. Parturients who suffered from heart disease or underwent cardiac surgery, are more vulnerable to hemodynamic disturbances during pregnancy and may develop decompensation of heart function or even acute heart failure due to increased cardiac workload during pregnancy. When heart failure is diagnosed, immediate termination of pregnancy or cesarean section with simultaneous cardiac surgery should be considered. These procedures will undoubtedly pose significant challenges for perioperative services, especially intraoperative management [12]. We should pay very close attention preoperatively to cardiac function and placental blood flow of the parturient, who may need delicate monitoring and adequate analgesia, to avoid severe fluctuation of blood pressure. Either epidural or general anesthesia can usually be the preferred method, but general anesthesia seems to be a better choice for patients with hemodynamic instability and potential bleeding during surgery. Beijing An Zhen Hospital and Wuhan Union Hospital of Tongji Medical College Huazhong University of Science and Technology have accumulated a wealth of experience in clinical anesthesia on parturient complicated with pulmonary hypertension, Eisenmenger syndrome, aortic dissection, and acute heart failure. They obtained very good outcomes and offered patients very favorable 
prognosis. Their experience can potentially be validated as recommendations for parturient with severe cardiac diseases undergoing obstetric or other surgical procedures.

\section{Intraoperative cell salvage in obstetric patients}

With the implementation of the two-child policy, the percentage of parturient with placental abnormalities and acute and severe cardiac diseases is increased, so is the risk of acute massive bleeding during cesarean section. Intraoperative cell salvage during obstetric procedures was generally not advocated due to traditionally-believed potential maternal-fetal antigen-antibody inter-reactions [6]. However, recent clinical studies have demonstrated that by adopting suction with double pipettes, adequate anticoagulation, sufficient washing and leukocyte filters, intraoperative cell salvage could be carried out and the blood can be returned to the pregnant woman safely, immensely reducing allogeneic blood transfusion requirement [6]. Currently some relevant clinical applications and research evaluations of intraoperative cell salvage in obstetric procedures have been ongoing, and it will potentially become one of the important supports for reducing allogenic blood transfusion in obstetric procedures.

\section{Application of labor analgesia}

In early 1951s, some physicians put forward a concept of painless childbirth, and they conducted a series of investigations such as walking labor analgesia and other related analgesic techniques for childbirth. They achieved encouraging results [13-15]. However, labor analgesia has been lagging behind in mainland China due to several factors, such as limited educational level of pregnant women and their families, low acceptance of obstetricians and midwives, insufficient medical resources in many areas, and lack of anesthesia providers who have the training and skills, yet have the time to provide the service [16]. In 2004, the People's Daily published an article entitled "Parturient enjoying painless labor less than $1 \%$-China to promote painless labor and delivery". Therefore, in consideration of the current status of high cesarean section rate and low labor analgesia rate in mainland China, Professor Lingqun $\mathrm{Hu}$ of Northwestern University and other overseas Chinese anesthesiologists launched "No Pain Labor \& Delivery-Global Health Initiative", a large public health project in $2006[16,17]$. At the same time, the Obstetric Anesthesia Group of CSA also initiated "Happy delivery to primary hospital and happy delivery room" project, aimed at implementation of safe and effective neuraxial labor analgesia, in an attempt to alter the 'lagging behind' status of labor analgesia in mainland China, and to ensure maternal, fetal and neonatal safety. After nearly a decade of aggressive promotion of labor analgesia, there have been plenty of domestic hospital systems to offer labor analgesia services. These efforts have greatly heightened hospital ad- ministration's awareness of labor analgesia, decreased the cesarean section rate, and consequently propelled the development of obstetric anesthesiology in mainland China.

\section{Fetal surgeries}

Fetal surgery refers to surgical intervention for congenital abnormalities of the fetus. It can be divided into minimally invasive surgery in the second trimester, open surgery in the second trimester, and intrapartum fetal surgery. Fetal surgery brings with it serious challenges on perioperative anesthetic management. In recent years, with the numerous breakthroughs in fetal surgeries, fetal anesthesia has also made huge progresses [5]. At present, several large hospitals in China have carried out fetal surgeries, and the operations are limited to intrapartum fetal surgeries. Meanwhile, valuable experience has been accumulated. Although great progress has been made in anesthetic management of fetal surgeries, many related problems such as the influence of narcotic drugs on fetal development have not been satisfactorily elucidated yet. The optimal anesthetic plans for fetal surgery still needs further investigations and modifications.

\section{Scientific research in obstetric anesthesia}

In recent years, Chinese obstetric anesthesia providers have conducted various basic and clinical studies. With rapid increase in number and level of academic articles published, more than 30 articles are covered by Science Citation Index (SCI)-indexed journals. These achievements have noticeably improved the situation of "Strong clinical aspect and weak scientific research" existing in mainland China for many years [9,18-21]. Although some of the basic research articles lack the in-depth exploration and originality of scientific investigation and some have controversial results and conclusions or may need further studies to validate their results, we do see hope for the scientific development of obstetric anesthesiology. We will encourage more young scientific minds to ponder the problems and controversies confronting current obstetric anesthesia and carry out more relevant basic researches. Thus, we will build a great reputation of Chinese obstetric anesthesiology in the world stage.

\section{High risk of obstetric anesthesia}

The two-child policy leads to an increased ratio of pregnant women with advanced maternal age and scarred uterus, which potentially cause dramatically high incidence of placenta previa, placental abruption and other obstetric bleeding complications. These will usher in more difficulties in anesthetic selection and perioperative management. The issue of selecting a delivery mode in pregnant women with scarred uterus can be very challenging for both pregnant women and medical professionals. The maternal obesity rate is also rising year after year. The "big" problem is 
that the larger maternal size is usually associated with higher incidence of hypertension and diabetes, increased difficulty in neuraxial anesthesia placement and tracheal intubation, and higher rate of fetal respiratory distress during childbirth. Besides, with assisted reproductive demands ever-increasing, gemellary and multiple pregnancy is also up-trending, resulting in higher rates of hypertension, anemia, premature delivery, postpartum hemorrhage, and other obstetric complications. This will eventually further complicate the perioperative management.

\section{New technology and ideas in obstetric anesthesiology}

We should strengthen preoperative management of pregnant women before anesthesia and delivery. Also, we will need multidisciplinary approach in dealing with high risk obstetric patients, teaming with obstetricians and other perioperative services to establish optimal delivery strategy and manage other various critical and severe co-existing medical conditions to minimize/decrease severe complications in the obstetric patient population. Also, we must reinforce delicate monitoring of patients in obstetric anesthesia. It is paramount to monitor vital signs of both mother and fetus in both labor analgesia and in cesarean section. We need to implement noninvasive or invasive hemodynamic monitoring to closely monitor preload and afterload, blood volume, coagulation function, Cardiac output and index, stroke volume variations and so on. In addition, we ought to actively adopt new technology in labor analgesia, to lower cesarean section rate and other obstetric complications. As long as the parturient have demands of labor analgesia and no contraindication, meticulous measures should be undertaken as soon as possible before the delivery mode is determined. Besides, we need to enhance obstetric emergency response capacity. For high risk obstetric patients, we should focus on improving our ability to perform a 5-minute emergency cesarean section and treatment of hemorrhagic shock. We need to keep ourselves abreast of current new progress in technology and management skills in obstetric anesthesia. We will adopt new technologies and new knowledge such as ultrasound assisted lumbar puncture technique in spinal/epidural anesthesia for gynecologic and obstetric procedures, antihypotensive drugs in spinal anesthesia, dural puncture epidural technique, visualization technique, intraoperative cell salvage in obstetric patients and anesthetic management in different fetal surgical approaches. We need to revise or update guidelines and specifications for obstetric anesthesia on regular basis according to national socioeconomic status and clinical and scientific discoveries. By doing all these, we will practically raise the level of standard care in obstetric patients in mainland China.

\section{Promoting anesthesiology as a profession by propagan- da, education and adequate staffing}

The shortage of anesthesiologists and other professional anesthesia providers is a huge problem that the Chinese anesthesiology community is currently facing. Many factors are contributing to this: the high intensity and high stress nature of the profession, the less visible nature among many other medical specialties, the inappropriate compensation level etc. This shortage has become a significant factor restricting the advancement of anesthesiology in mainland China. Therefore, understanding the forthcoming upsurge in demand of obstetric anesthetic care obstetrics and more complex challenges, we should improve publicity and visibility of anesthesiology as a medical profession, train adequate anesthesia personnel to adequately staff the obstetric anesthetic care to meet the demand and challenges from obstetric care and the development obstetric anesthesiology.

Obstetric anesthesiology is a subspeciality involving multidisciplinary approach. Hospital administration and obstetricians need to be more aware of the importance of obstetric anesthesia, encouraging the parturient to have labor analgesia. Building a multidisciplinary team to manage high risk obstetric patients, the comprehensive management team will be patient care-centered. Obstetric anesthesiology will need to adopt new technology to optimize the process and specifications of obstetric anesthesia and labor analgesia. By all these efforts, obstetric anesthesiology will significantly improve the standard of care for obstetric patients, and the development of obstetric anesthesiology in mainland China will be fast-moving forward.

\section{Acknowledgements}

\section{None}

Disclosure of Funding: This work is supported by the grants from the National Natural Science Foundation of China (Grant \# 81671890).

Conflict Interests Disclosure: The authors have no conflicting interests to disclose.

Corresponding Author: Shanglong Yao, MD, PhD; Professor, Department of Anesthesiology, Institute of Anesthesiology and Critical Care Medicine, Union Hospital, Tongji Medical College, Huazhong University of Science and Technology, No. 1277 Jiefang avenue

Wuhan, Hubei 430022, PR China. Phone: +8613886128437,Email: ysltian@163.com

Editor: Jun-Ming Zhang, MD, MSc, Pain Research Center, Department of Anesthesiology, University of Cincinnati College of Medicine. 231 
Albert Sabin Way, Cincinnati, OH 45267-0531, Phone:+1-513-558-2427 Fax: +1-513-558-0995, Email: jun-ming.zhang@uc.edu

\section{Additional publication details}

Journal short name: Transl Perioper \& Pain Med

Received Date: June 1, 2017

Accepted Date: July 1, 2017

Published Date: July 18, 2017

Transl Perioper \& Pain Med 2017; 2 (3):23-28

\section{Citation and Copyright}

Citation: Lisha Hu.Shanglong Yao. The development of obstetric anesthesiology in mainland China. Transl Perioper \& Pain Med 2017; 2(3): 23-28

Copyright: (C) 2017. This is an open-access article distributed under the terms of the Creative Commons Attribution License, which permits unrestricted use, distribution, and reproduction in any medium, provided the original author and source are credited.

\section{References}

1. Caton D. The History of Obstetric Anesthesia. In: David H. Chestnut LSP, Lawrence C. Tsen, Cynthia A. Wong, ed. Chestnut's obstetric anesthesia: principles and practice. 4th ed. Philadelphia: Elsevier Saunders: p3-12. 2014.

2. Feng BB, Wang L, Zhai JJ. Investigation on delivery analgesia effect of combined spinal epidural anesthesia plus Doula and safety of mother and baby. Clin Exp Obstet Gynecol. 2013; 40:574-8. PMID: 24597260

3. Li B, Wang H, Gao C. Bupivacaine in combination with fentanyl or sufentanil in epidural/intrathecal analgesia for labor: a meta-analysis. J clin pharmacol. 2015; 55:584-91. PMID: 25535687

4. Li Z, Tian M, Zhang C, Li A, Huang A et al. A Randomized Controlled Trial to Evaluate the Effectiveness of Intrathecal Bupivacaine Combined with Different Adjuvants (Fentanyl, Clonidine and Dexmedetomidine) in Caesarean Section. Drug Res (Stuttg). 2015; 65:581. PMID: 25504002

5. Wu JL, Deng DR. Selection of anesthesia methods for intrapartum fetal operation. J Practic Ob Gyn. 2016; $32: 415-7$.

6. Xing N, Peng YS, Wei X, Du YY, Zhang W. Literature analysis on feasibility studies of intraoperative cell salvage in Caesarean. Chin J Anesth. 2015; 35:858-61.

7. Chen SF, Zhang C, Chen Y, Cheng WW. Analysis of factors influencing safety of department of obstetrics based on "the second child" policy and investigation of countermeasures. J Shanghai Jiaotong Univ Med Sci. 2016; 36:742-6.

8. Lin DM, Lu JK. Anesthetic management in pregnant patients with severe idiopathic pulmonary arterial hypertension. Intl J Ob Anesth. 2014; 23:289-90. PMID: 24910350

9. Tang WX, Li JJ, Bu HM, Fu ZJ. Spinal anesthesia with low-dose bupivacaine in marginally hyperbaric solutions for caesarean section: A randomized controlled trial. Eur J Anaesth. 2015; 32:493. PMID: 25032943

10. Yang JL, Zhang LS. The international fashion trend for obstetric anesthesia administration_ obstetric anesthesia: epidural, spinal or combined spinal-epidural analgesia. Int J Anesth \& Resusc. 2013; 34:289-92.

11. McGinn B, Zhou J. Physiologic changes during pregnancy. In: Linda S. Aglio RWL, Richard D. Urman, ed. Essential Clinical Anesthesia Review: Keywords, Questions and Answers for the Boards Cambridge: Cambridge University Press:p. 382-4. 2015.

12. Guidelines for the diagnosis and treatment of hypertensive disorders in pregnancy. Chin J Ob Gyn. 2015; 50:7218.

13. Preliminary observation of acupuncture for painless labor. J Traditional Chin Med. 1959:53.

14. Liu H, Zhou J, Tang Y, Ma Y, Xie C et al. Survey of labor epidural analgesia and patient education for women in southwest China. J Anesth. 2012; 26:800-1. PMID: 22584818

15. Zhang LC, Tian GG, Zhu JZ. Study on epidural walking labor analgesia with extremely low concentration ropivacaine combined with sufentanil. Maternal \& Child Health Care China. 2013; 28:3669-71

16. Hu LQ, Li YP, Xia Y, Tao WK, Zhao PS. From "No Pain Labor \& Delivery-Global Health Initiative" to see China's labor analgesia. J Clin Anesth. 2013; 29:205-8.

17. Hu LQ, Flood P, Li Y, Tao W, Zhao P et al. No Pain Labor \& Delivery: A Global Health Initiative's Impact on Clinical Outcomes in China. Anesth Analg. 2016; 122:1931. PMID: 27195636

18. Zhou G, Zhang X, Ge S. Retrospective analysis of acute fatty liver of pregnancy: twenty-eight cases and discus- 
sion of anesthesia. Gynecol Obsten Invest. 2013;76:83-9.

PMID: 23796980

19. Wang LZ, Zhang YF, Hu XX, Chang XY. A randomized comparison of onset of anesthesia between spinal bupivacaine $5 \mathrm{mg}$ with immediate epidural $2 \%$ lidocaine $5 \mathrm{~mL}$ and bupivacaine $10 \mathrm{mg}$ for cesarean delivery. Int J Obstet Anesth. 2014 Feb; 23(1):40-4. PMID: 24200700

20. Liu X, Xue A. A randomized trial of remifentanil for analgesia in external cephalic version for breech presentation. Medicine. 2016; 95:e5483. PMID: 27930530

21. Liu Q, Wang JJ, Yan HY, Wang YQ, Qin CW et al. Safety and pharmacodynamic mechanism of ropivacaine lumbar anesthesia in Cesarean section. J Biol Regul Homeost Agents. 2017 Jan-Mar; 31(1):71-76. PMID: 28337872 